\title{
Age-related differences in dynamic balance control during stair descent and effect of varying step geometry
}

\author{
A.C. Novak, V. Komisar, B.E. Maki, G.R. Fernie \\ Version Post-print/accepted manuscript \\ Citation Novak, A. C., Komisar, V., Maki, B. E., \& Fernie, G. R. (2016). Age- \\ (published version) related differences in dynamic balance control during stair descent \\ and effect of varying step geometry. Appl Ergon, 52, 275-284 \\ Publisher's Statement

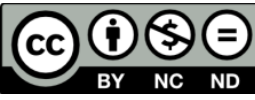 \\ (C) <2016>. This manuscript version is made \\ available under the CC-BY-NC-ND 4.0 license \\ http://creativecommons.org/licenses/by-nc-nd/4.0/
}

Always cite the published version, so the author(s) will receive recognition through services that track citation counts, e.g. Scopus. If you need to cite the page number of the TSpace version (original manuscript or accepted manuscript) because you cannot access the published version, then cite the TSpace version in addition to the published version using the permanent URI (handle) found on the record page. 
Age-related differences in dynamic balance control during stair descent and effect of varying step geometry

\author{
Novak AC ${ }^{\mathrm{a}}$, Komisar $\mathrm{V}^{\mathrm{a}, \mathrm{b}}$, Maki BE $\mathrm{BE}^{\mathrm{a}, \mathrm{b}, \mathrm{c}, \mathrm{d}}$, Fernie GR $^{\mathrm{a}, \mathrm{b}, \mathrm{c}, \mathrm{d}}$
}

aiDAPT, Toronto Rehabilitation Institute - University Health Network, 550 University Ave, Toronto, Ontario, M5G 2A2 Canada, www.cealidapt.com

${ }^{\mathrm{b}}$ Institute of Biomaterials and Biomedical Engineering, University of Toronto, 164 College Street, Toronto, Ontario, M5S 3G9, Canada

${ }^{\mathrm{c}}$ Department of Surgery, University of Toronto, 149 College Street, Toronto, Ontario, M5T 1P5, Canada

dInstitute of Medical Science, University of Toronto, 1 King's College Circle, Toronto, Ontario, M5S 1A8, Canada

Tables: 2

Figures: 6

\title{
Corresponding Author:
}

Dr. Alison C. Novak

iDAPT (www.cealidapt.com), Toronto Rehabilitation Institute - University Health Network 550 University Ave, Toronto, Ontario

M5G 2A2 Canada

Email: alison.novak@uhn.ca

Tel: 416-597-3422 x7713

Fax: 416-597-3027 


\begin{abstract}
The incidence of stairway falls and related injuries remains persistently high; however, the risk of stair injuries could be reduced through improved stairway design. The current study investigated dynamic balance control during stair descent and the effects of varying the step geometry. Data were collected from 20 healthy young and 20 older adults as they descended three staircases (riser heights of 7, 7.5 and 8 inches (178, 190 and $203 \mathrm{~mm}$, respectively)). At each riser height, the tread run length was varied between 8 and 14 inches $(203 \mathrm{~mm}$ and 356 $\mathrm{mm})$ in one-inch $(25 \mathrm{~mm})$ increments. Kinematic data provided measures of segmental and whole-body dynamic control. Results demonstrated that older adults had greater lateral tilt of the upper body than young adults, but actually had larger margins of stability than the young in the antero-posterior direction as a result of their slower cadence. Nonetheless, for both age groups, the longer run lengths were found to provide the largest margins of stability. In addition, increase in run length and decrease in riser height tended to reduce forward upper body tilt. These results help to explain the underlying biomechanical factors associated with increased risk of falls and the relationship with step geometry. Considering the importance of stair ambulation in maintaining independence and activity in the community, this study highlights the definite need for safer stair design standards to minimize the risk of falls and increase stair safety across the lifespan.
\end{abstract}

\title{
Keywords
}

Stair ambulation, kinematics, dynamic balance, aging 


\section{Introduction}

Each year, approximately one out of three older adults will experience a fall and of those, approximately $50 \%$ will experience repeated falls [1]. Additionally, over $50 \%$ of older adults who have previously fallen will also develop a fear of falling [2-4]. This fear leads individuals to avoid many fundamental activities of daily living, contributing to decline in physical function, independence, level of activity and health-related quality of life [4-6]. Although a fall can occur in many types of natural and built environments, research consistently identifies stairs as a primary location of serious fall-related injury [7], underscoring stairs as one of the most hazardous locations of any home $[8,9]$. Serious falls on stairs can happen across the lifespan; however, they are most prevalent in advanced age, when the consequences are most likely to be injurious, disabling or fatal [7]. For an older adult, a stair-related fall can lead to hospitalization, admission to a longterm care home, or death $[7,9]$.

Stair negotiation is a complex locomotor task that imposes significant challenges to movement control in persons of all ages [10-18]. Compared to level walking, stair ambulation demands greater balance control. Stair descent is a particularly dynamic process compared to ascent $[7,16]$, as descending stairs involves the forward acceleration of the large upper body mass with each step. This forward motion must be arrested before a toppling occurs by applying a sufficient moment in the legs or through the handrail to counteract the movement. Greater inherent instability during descent has been indicated by larger maximum divergence of the centre of mass (COM) beyond the centre of foot pressure (COP) [18] as well as faster COP velocity when compared to ascent [16]. Age-related differences in gaze behaviour during stair descent have also demonstrated that older adults spend more time looking at the next stair tread [19], and it has been hypothesized that this behaviour may be associated with a forward flexed 
position of the upper body and an anterior shift of the body's COM during stair descent [10]. More recently, assessment of the margin of stability during stair descent has indicated that older adults are at a greater risk of falls during stair descent than young adults due to a reduced ability to safely control the body's COM [10]. This is mainly a result of higher velocity of the COM leading to a more anterior position of the vertical projection of the COM. Bosse and colleagues [10] have also shown a significant correlation of COM velocity with joint angular impulse of the ankle and knee extensors, indicating that a greater amount of mechanical work is required to arrest the motion. This suggests that age-related declines in lower limb strength and muscle power $[13,20]$ may limit the ability of older adults to safely control the body's COM while walking down stairs, and places them at greater risk of falls than young adults during stair descent.

It has been emphasized that the risk of stair injuries can be substantially reduced by improved stair design $[8,21]$, which includes features such as riser height (the vertical distance from one step to the next), nosings (horizontal protrusion beyond the edge of the run) and tread run (the horizontal distance between adjacent nosings or in the absence of a step nosing, between the edges of adjacent steps) (Figure 1). For example, a large injury surveillance study in the United Kingdom estimated that increasing the minimum run length several inches could reduce stair-related falls by a factor of four [22]. Despite the available evidence, there is still debate amongst decision makers regarding the appropriate step geometry design to minimize falls during stair ambulation. This is likely attributable to the lack of comprehensive studies aimed at understanding step dimensions and falls. In the few studies that have investigated the role of step geometry, the focus has been limited to studying the effect of varying step geometry on foot placement, energy costs or joint demands during stair ascent and descent [8, 23-25], with no 
inclusion of older adults or investigation of balance. For example, to support recommendations for safer riser heights and run lengths, Wright and Roys [23] reported that with runs of $300 \mathrm{~mm}$ and above, a greater proportion of the foot is placed on the step. Higher risers also results in forward placement of the foot on the tread, regardless of the length of the step, suggesting an optimal riser height of $190 \mathrm{~mm}$ or less to minimize risk of the foot slipping/pivoting off the step [23]. Step geometry must facilitate gait and adequate footing. However, the minimum recommendations to support safe stair ambulation are difficult to provide without understanding the impact of step geometry on one's ability to also maintain balance. Although it has been shown that a steeper stair results in large variability in vertical loading of the stance leg during descent, leading to a less stable gait pattern [26], no study has directly investigated dynamic balance control related to variations in step geometry and the effects of age. The lack of comprehensive studies investigating the effect of stair geometry is likely the reason that stair standards internationally vary to such a great extent at the possible expense of safety. For example in Canadian homes, the minimum run length is $210 \mathrm{~mm}$ and maximum riser height is $200 \mathrm{~mm}$, whereas the ICC's (International Code Council) International Residential Code stipulates a minimum run length of $255 \mathrm{~mm}$ and maximum riser height of $196 \mathrm{~mm}$.

Stair descent is unique compared to other activities of daily living due to the constraints on the base of support (BOS). Specifically, since an adult's forefoot placement will typically extend beyond the edge of the stair tread [23], the anterior BOS limit is often defined by the edge of the tread. It follows that a positive change to the BOS (ie. by increasing the length of the step run) would permit greater margins of stability in the older group without the need to alter individual behaviour. In addition, a reduction in the riser height is expected to reduce variability of movement (as has been shown with vertical loading [26]), suggesting improved stability during 
stair descent. The purpose of the current study was to investigate age-related differences in dynamic balance control during stair descent and the effect of varying step geometry, i.e. riser height and run length.

\section{Material and Methods}

\subsection{Participants}

Twenty healthy young adults (YA) (18 to 30 years old) and 20 healthy older adults (OA) (over 65 years old) were recruited for the study. However, analysis was restricted to 14 of the 20 YA and 14 of the $20 \mathrm{OA}$ (six participants per group were excluded due to problems with the data acquisition system). A priori sample size calculation was completed assuming a moderate effect size (.25) to analyze one between-subject factor (age group) and two within-subject factors (riser height and run length), and assumed a very conservative correlation of .25 among repeated measures. This yielded a total sample of 26 participants to achieve a high level of power (.8). Since we collected data from 20 participants per group, with analysis performed on 14 participants per group, our sample size criteria were met. All participants were right-leg dominant. Participant characteristics are presented in Table 1.

\section{[INSERT TABLE 1]}

Participants were recruited from the local community through posted advertisements and the research institute's central recruitment database. Inclusion criteria required that all participants could ascend and descend stairs independently without the use of a handrail, were in self-reported good health, and had normal or corrected-to-normal vision (self-report). 
Participants were excluded from the study if they presented with any neurological or orthopaedic condition affecting walking ability.

\subsection{Staircase apparatus}

Three customized, freestanding staircases were built for the testing protocol (Figure 1). Each staircase consisted of 6 steps with run lengths that could be adjusted between 8 and 14 inches. The riser height for each staircase was constant ( 7 inches, 7.5 inches, and 8 inches, respectively). All corresponding metric measurements for the rise and run step dimensions tested are presented in Table 2. Handrails were present on the right side for all testing. However, participants were instructed to descend the stairs without the use of the handrail.

\section{[INSERT TABLE 2]}

\subsection{Subject instrumentation and procedure}

Data collection took place at Toronto Rehab Institute's Challenging Environments Assessment Laboratory (www.cealidapt.com). All testing took place during a single testing session, lasting approximately 2 hours. Ethics approval was provided by the institution's ethics board and all participants provided written informed consent prior to participation in the study.

Three-dimensional biomechanical data were acquired during the task using 3 optoelectric camera banks (VZ4000, Phoenix Technologies Inc., Burnaby, BC) placed in front of the staircases. To quantify segmental trunk motion and measures of dynamic balance, infraredemitting diodes (IREDs) were secured at the sacrum (level of S2, providing a proxy measure of whole body COM location [27]) and the upper body (at the thoracic level of T12). A rigid cluster 
of three IRED markers was secured to each foot. An instrumented probe was used to identify the distal and proximal ends of the foot and ankle (virtual landmarks) with respect to the tracking markers. The motion capture markers on the probe were positioned at a fixed location with respect to the tip, which allowed the spatial coordinates of each virtual landmark to be calculated by transforming the IREDs' location to the tip of the probe.

Two IRED markers were also secured on the riser face of each step for each staircase, away from the travel path of the subject. The instrumented probe was used to identify the edges of the stair treads in the global coordinate system relative to the markers on the stair risers.

Following instrumentation, participants were instructed to ascend and descend the stairs at a self-selected pace, under normal lighting conditions. Participants wore their own footwear (comfortable walking or running shoes) for testing. For this study, only stair descent is reported. All participants were instructed to complete two trials for each staircase configuration while performing a secondary cognitive task, where the participants were asked to count backwards out loud by 3's (starting with a randomly selected number) from the initiation to completion of the stair walking task. Previous dual-task studies have shown that the addition of a secondary cognitive task reveals more natural motor behaviour patterns, thus minimizing any associated effects of laboratory testing [28]. A total of 21 conditions were tested ( 7 run lengths $\mathrm{x} 3$ riser heights). The step geometry configurations were randomized to ensure that fatigue, practice effects or carry-over effects did not affect interpretation of the data. An overhead, passive safety harness system was used for all testing, designed so as to not interfere with participants' normal gait.

\subsection{Data Analysis}


All kinematic data were collected at $60 \mathrm{~Hz}$, filtered using a second-order, zero-lag low-pass Butterworth filter (cut-off frequency $=6 \mathrm{~Hz}$ ), interpolated (cubic spline interpolation, maximum missing gap of 10 frames or less) and processed using Visual 3D (C-Motion, Inc.). Variables of interest were determined for the first transition step (step 1, where the individual transitions from level walking to stair descent) and for steady state stair descent (considered as the middle step 3) [29]. During stair descent, the transition step can be particularly challenging as the individual must adjust their locomotive pattern to accommodate the change in task demands [29], and is reported as a common location for missteps and accidents [25]. During steady state stair descent, progression velocity reaches a steady state [29] and movement is refined [11].

In order to quantify measures of dynamic balance, several measures were determined. The horizontal distance between the COM projection and the step edge within the sagittal plane (dCOM) was determined for the transition step and steady state step. The margin of stability was also calculated [10] in the sagittal plane. This was computed as the instantaneous distance between the extrapolated COM (XCOM) and the anterior boundary of the base of support (defined by the edge of the step when the foot was placed beyond the step edge). When the foot was placed entirely on the step with no foot overhang, the anterior boundary of the base of support was defined by the front of the foot. In a static situation, the minimum distance from the COM location to the boundary of the base of support defines the margin of stability. However, in a dynamic situation, the destabilizing effect of the COM motion also has to be taken into account. The extrapolated COM accomplishes this by including a term $\left(\mathrm{vCOM} / \sqrt{ }\left(\mathrm{gl}^{-1}\right)\right)$ that represents the COM displacement that would be required to arrest the COM motion, as determined using the well-established inverted pendulum model [30]. A positive margin of stability indicates postural stability (ie. the XCOM is controlled within the base of support) 
whereas a negative margin of stability indicates the XCOM exceeds the boundaries of the base of support [10].

As previously described $[10,28]$, the XCOM is determined as follows:

$$
\mathrm{XCOM}=\mathrm{pCOM}+\mathrm{vCOM} / \sqrt{ }\left(\mathrm{gl}^{-1}\right)
$$

Where, $\mathrm{pCOM}=$ anterior-posterior component of the vertical projection of the COM in the global coordinate system

$\mathrm{vCOM}=$ anterior-posterior velocity of the COM

$\mathrm{g}=$ acceleration due to gravity

$1=$ distance between the COM and the ankle

In addition to the margin of stability, sagittal and frontal plane angles of the upper body (upper body tilt angle in the forward-backward and medial-lateral directions, respectively) were also determined with respect to standing neutral posture using the markers secured at T12 and S2. Many falls during stair descent are thought to be precipitated by errors during the foot landing (e.g. oversteps); hence, COM dynamics and trunk orientation are likely to be crucial at this point in time. The upper body represents more than $50 \%$ of the total body mass, which must be controlled during stair descent. In the event of an overstep, an increased forward tilt of the upper body (or increased flexion of the trunk) increases the risk of a fall. If the upper body is placed further forward, this may challenge one's ability to counter the forward momentum of the upper body. All measures of interest were thus determined at the onset of foot contact, for both the first transition step and for a step (step \#3) during steady state stair descent.

\subsection{Statistical Analysis}

Descriptive statistics (means, standard deviations) were included for all primary variables of interest (upper body tilt angles, margin of stability) and secondary variables of interest 
(dCOM, vCOM). Statistical analyses involved repeated-measures ANOVA, and were performed using SAS 9.4 PROC MIXED (SAS version 9.4, Cary, NC). Fixed effects were rise, run and age group. Subject was specified as a random effect, with age group nested within. Parameters were estimated using the method of restricted maximum likelihood (REML). The full model included all parameters with all third-order interactions (rise-by-run, run-by-age, rise-by-age, rise-by-runby-age). However, no rise-by-run-by-age interactions were found and therefore the reduced model included only second-order interactions. Post-hoc pairwise comparisons were conducted following the identification of a significant main effect, using Tukey's HSD adjustments to account for multiple comparisons. A significance level of $\mathrm{p}<.05$ was adopted for all analyses.

\section{Results}

\subsection{Segmental control: Forward and lateral upper body tilt angles}

Figure 2 demonstrates the general relationship of the run length (A) and riser height (B) and the forward upper body tilt angle across age groups. No significant main effect of age was detected for forward upper body tilt angle for both steady state $(\mathrm{p}=.22)$ and transition steps $(\mathrm{p}=.1)$

Run length had a significant main effect on forward upper body tilt angle for both the steady state step and the transition step (p's<.0001), and the effects tended to be similar at all three riser heights (run-by-rise interaction, p's>.07) and for both age groups (run-by-age interaction, $\mathrm{p}$ 's $>.22$ ). In pairwise comparisons, both OA and YA demonstrated a significant increase in the forward upper body tilt angle at shorter run lengths. Specifically, the forward upper body tilt angle for both transition and steady state steps at the shortest run length (8 inches) were significantly greater compared to all other run lengths ( $\left.\mathrm{p}^{\prime} \mathrm{s}<.0001\right)$. In addition, as the run 
length increased to 9 inches, the forward upper body tilt angle was significantly higher in comparison to run lengths that were 12 inches and longer ( $\left.\mathrm{p}^{\prime} \mathrm{s}<.03\right)$. For the steady state step, the 10 inch and 11-inch runs also resulted in significantly higher forward upper body tilt angles compared to 12 and 14 inches ( $\left.\mathrm{p}^{\prime} \mathrm{s}<.02\right)$. In contrast, for the transition step, no significant pairwise comparisons were detected for run lengths comparing runs 11 inches or longer ( $p>.06)$.

Riser height had a significant main effect on forward upper body tilt angle during the steady state steps $(\mathrm{p}=.03)$ but not during the transition steps $(\mathrm{p}=.27)$. For the steady state step, post hoc pairwise comparisons revealed a significant reduction in forward upper body tilt angle with 7-inch risers compared to 8-inch risers. However, a significant riser-by-age interaction (for both transition step, $\mathrm{p}<.02$, and steady state step, $\mathrm{p}<.01)$ revealed that the effects of riser height were limited to the OA. Within the OA, forward upper body tilt angle was approximately $46 \%$ and $26 \%$ smaller for the 7 -inch riser compared to the 8 -inch riser, for transition and steady state steps respectively.

Step geometry (run length, riser height) did not impact lateral upper-body tilt angle. However, a significant age effect was found, where OA demonstrated significantly greater lateral upper body tilt angle compared to YA $(\sim 32 \%$ greater $)$ during the steady state step $(\mathrm{p}=.03)$ (Figure 3).

[INSERT FIGURE 2]

[INSERT FIGURE 3]

\subsection{Margin of stability}


At the onset of foot contact for the steady state step, older adults demonstrate increased margins of stability compared to young adults $(\mathrm{p}=.05)$ (Figure 4). Similar age-related effects on margin of stability were not detected for the transition step $(\mathrm{p}=.11)$. A main effect of run length was detected for the margin of stability for both the transition step $\left(p^{\prime}<^{<.002)}\right.$ and steady state step $\left(p^{\prime} s<.005\right)$. No significant rise-by-run $(p=.38)$ or age-by-run interactions were found for the steady state step ( $\mathrm{p}=.92)$. Following pairwise post hoc comparisons for run length, 9 and 10 inch run lengths resulted in reduced margins of stability compared to 13 and 14 inch run lengths (steady state step; $\mathrm{p}<.007$ ), on the transition step, the margins of stability were lower for 10 and 11 inch run lengths compared to the 14 inch run length $(\mathrm{p}<.03)$. The observed reductions in margin of stability at shorter run lengths cannot be attributed to changes in COM velocity, which tended to increase with increased run lengths $(\mathrm{p}<.0001$; Figure 5). Instead, it appears that the reduced margin of stability at the 9 and 10 inch runs was primarily due to changes in COM displacement, as both age groups demonstrated reduced distance between the COM and the stair edge for the 8, 9 and 10 inch run lengths compared to 11 inch runs and longer ( $\mathrm{p}<.001$; Figure 6).

A significant main effect of riser height was also detected for the margin of stability (p's<.005). This effect was similar across both age groups (no significant rise-by-age interaction; p>.33)). The 7-inch riser resulted in reduced margins of stability compared to 7.5-inch and 8inch risers for the steady state step $(\mathrm{p}<.01)$. For the transition step, the 7 -inch riser had reduced margins of stability compared to the 7.5-inch risers $(\mathrm{p}=.009)$, but not when compared to the 8inch risers $(\mathrm{p}=.41)$. The differences in margin of stability correspond to a riser-height effect in the pCOM, as both young and older adults exhibited increased distance between the body's COM and the step edge on the 7.5-inch riser compared to the 7-inch riser for the steady state step 
$(\mathrm{p}=.003)$ and the transition step $(\mathrm{p}<.0001)$. The vCOM component of the margin of stability was not significantly influenced by riser height ( $>$ >.29).

[INSERT FIGURE 4]

[INSERT FIGURE 5]

[INSERT FIGURE 6]

\section{Discussion}

This study aimed to examine dynamic balance control in healthy young and older adults and the effect of changes in step geometry. Results demonstrated that older adults had greater lateral upper body tilt angles during stair descent than young adults, but actually had larger margins of stability at foot contact than the young adults in the antero-posterior direction as a result of their slower gait. Nonetheless, our results indicate that specific changes in the run length and riser height can have clear benefits to maintaining balance during stair descent, for both age groups. Specifically, longer run lengths were found to provide the largest margins of stability, as determined by a model that takes into account both the position and velocity of the COM [30]. In addition, an increase in run length and decrease in riser height tended to reduce forward pitch of the upper body. Considering the importance of stair ambulation in our daily lives and the increased risk associated with descending stairs, small changes in step geometry can reduce the 
risk of falling. These results can be used to advocate for changes in codes and standards to improve stair safety.

Ergonomics-based research emphasizes that stair injuries can be prevented by improved stair design [8, 21-23], where undersized tread runs are often highlighted as a major problem in stairway safety [31, 32]. A United Kingdom study demonstrated that by increasing the minimum run length to at least 10 inches $(254 \mathrm{~mm})$, stair falls could be reduced 4-fold [22]. The results of the present study provide insight into the underlying mechanisms for this reduction in risk during stair descent from a dynamic balance control perspective. In particular, individuals were found to descend stairs with a reduced forward upper body tilt angles as the run length was increased. At the longer run lengths, the adopted posture resulted in greater distance between the body's centre of mass and the anterior boundary of the base of support and thereby provided greater stability margins despite increases in COM velocity.

The margin of stability represents the instantaneous difference between the base of support boundary and the extrapolated COM location, which adjusts the actual COM position to account for the COM displacement required to arrest the COM velocity [10]. Previous research investigating the margin of stability during stair descent on a limited-riser staircase ( 2 steps only) has shown that older adults are at a greater risk of falls compared to young adults, due in part to a diminished ability to generate extensor muscle output $[13,34]$ and safely control the body's COM $[10,35]$. We have shown that young adults actually exhibit greater instability according to the margin of stability compared to the older group. This is likely due to a combination of factors, including the increased COM velocity and increased cadence of the younger group (YA $=101.0+/-31.7$ steps $/$ minute; OA $=80.6+/-28.7$ steps $/$ minute; $\mathrm{p}=.05)$, both reflective of "riskier" or more dynamic behaviour. Although we did not measure physiological capacities, 
previous research has shown that young adults demonstrate increased strength and faster movement control compared to older adults during stair descent [34], which helps to explain why young adults are able to accommodate the greater negative margins of stability without falling. We also asked individuals to descend a 6-step staircase (rather than a 2-step staircase in the work by Bosse et al. [10]) and as a result the challenge of the task is greater, likely resulting in adaptive behaviours to maximize safety for our older group $[10,14,16]$. The reduced cadence observed in the older adults may represent one such an adaptation, and presumably this occurs to compensate for the diminished ability to safely control the body's COM. Of note, for both older and young adults, a negative margin of stability generally occurred at foot contact. As has been previously described, this is necessary to transition to the next step in an energetically efficient manner; however this is achieved at a cost to dynamic stability [10].

The margin of stability is dependent on many variables, including the limits of the base of support. During stair walking, the base of support is restricted to the edge of the steps. In home stairs, the run length of the step can be quite small (in Canada, for example, the minimum run length in the National Building Code is defined at $210 \mathrm{~mm}$ or $8 \mathrm{1} / 4$ inches). Providing an increased base of support during stair descent is only possible by increasing the run length. Analysis revealed that greater margins of stability occurred at the longer run lengths (13 and 14 inches), despite increased COM velocity. With shorter run lengths, stability is challenged because the distance between the anterior position of the body's COM relative to the anterior boundary of the base of support is reduced. At foot contact, one must control the forward momentum generated by the forward pitching motion of the upper body and COM, which becomes more difficult with aging. Previous work has shown that in response to a trip, agerelated decreases in reaction time will lengthen the time required to generate the moment 
required to arrest trunk angular velocity [36]. If one has a greater distance between the edge of support and the COM, such as is the case with a larger run length, a greater mechanical advantage is provided for the individual to generate adequate moments to counter forward falling motion. Indeed, in the older group, the longest run lengths actually provided positive margins of stability at foot contact during steady state stair descent.

Our results have shown that step riser height is also important. In particular, older adults increased the forward upper body tilt angle with higher risers compared to the lower risers. Somewhat surprisingly, however, the lowest riser height did not result in the largest margins of stability. Similar to the findings with respect to run length, the position of the centre of mass relative to the step edge was the main contributing factor to the stability margin differences (rather than the COM velocity). However, considering the very small differences (mean difference in stability margin of $1.4 \mathrm{~cm}$, in comparison to mean differences as large as $3.1 \mathrm{~cm}$ due to changes in run length), the result should be interpreted with caution. Further work is needed to investigate the relationship between riser height and balance control.

The forward upper body tilt angle findings are important because the upper body represents a substantial portion of the total body mass, which must be controlled during stair descent. In the event of an overstep, an increased forward upper body tilt angle (or increased flexion of the trunk) increases the risk of fall. This is particularly important for older adults, in light of the significant age-related changes in neuromuscular function which would challenge one's ability to counter the forward momentum of the upper body when it is placed further forward due to trunk flexion. Such alterations in upper body sagittal plane angles have also been shown for other gaitrelated tasks such as obstacle crossing [33] and have been suggested to increase risk of falls. Based on our findings, providing longer runs or shorter risers results in reduced upper body 
angles in the plane of progression allowing for a reduction in the risk without the need to alter individual behaviour patterns.

In addition to age-related changes in dynamic balance control in the sagittal plane, it has been previously shown that older adults experience greater hip and pelvis motion in the frontal plane during stair descent, in comparison to young adults [12, 13, 37]. This suggests that movement control deteriorates in the frontal plane with age $[38,39]$ and greater stabilization is required to compensate for these medial-lateral movements. The present findings also showed significant age-related differences in medial-lateral stability, as evidenced by increased lateral tilt of the upper body in the older adults. Moreover, this apparently represents a challenge of stair descent for older adults that cannot be mitigated through safer step design, as lateral upper-body tilt did not change across the tested range of riser height and run length dimensions (which represent commonly-used stair geometries). To the authors' knowledge, no study has reported frontal plane motion in relation to changes in step run length. One study [40] has shown that increases in step height (three heights tested: $7.2 \mathrm{~cm}$ (2.8 inches), $14.4 \mathrm{~cm}$ (5.7 inches), and 21.6 $\mathrm{cm}$ (8.5 inches)) significantly contribute to reductions in medial-lateral dynamic stability for older adults, as measured by RMS fluctuations in COM position in the frontal plane. The large range between the tested step heights likely contributes to the reported differences in RMS fluctuations of the COM. However, similar to our findings, Buckley et al. [40] reported no effect of step height as measured by the average COM-COP difference. Current home stair standards advocate for a maximum riser height of $17.8 \mathrm{~cm}$ (or 7 inches), although in Canadian homes 20.3 cm (8 inches) riser heights are permitted. [31] That "safer" step geometry does not alter mediallateral sway emphasizes the need for alternative compensatory tools to assist with stair descent, particularly for older adults given concomitant declines in muscle strength. This data underscores 
the importance of properly designed handrails, for example, to control medial-lateral sway and counter additional movement in the frontal plane [16].

\section{Limitations}

The present study is subject to a number of potential limitations. First, our analysis primarily focused on dynamic balance in the sagittal plane of movement. While stair-related falls generally occur in the anterior direction during stair descent, differences in frontal plane angles of the upper body (or lateral upper body tilt angles) reveal age-related effects in stair descent behaviour not affected by changes in step geometry. Future work should build on our findings associated with sagittal-plane stability and include a more comprehensive analysis of frontalplane stability during both stair ascent and descent. Considering persons with mobility impairment and greater reliance on hip adductors to assist with step clearance [41], it remains unknown whether step geometry plays an important role in accommodating lateral balance. Second, we asked participants to descend a 6-step flight of stairs, restricting our analyses to the initiation of foot contact for the first transition step and a single steady-state step. During normal daily life situations, many staircases will have a larger number of steps, and while our results yielded significant insight to the effect of step geometry on balance control, future investigation should consider the other critical phases of the stair gait cycle with a longer flight of stairs (for example, 13 steps which are common in home staircases).

It is important to note that recommendations for standards should consider the variability of the population. In terms of height, the mean participant height in this study reflects that of the Canadian population (Statistics Canada, 2015) which provides confidence that our data can be useful for guiding future recommendations. Although providing concrete recommendations for 
optimum stair design is beyond the scope of this paper since we do not consider persons with mobility impairment, we certainly provide a greater understanding of the biomechanical risk factors of falls and the relationship with step geometry. Future work will include a greater range persons with and without mobility limitations to guide recommendations. Of note, we did not control for anthropometric dimensions of our tested sample, nor did we normalize the data according to height or weight of our participants. Although the size of our sample reflects the general population, such anthropometric variables certainly could have an effect on the findings, and should be considered in future analyses of stair ambulation.

Finally, due to limitations with our instrument set-up, we were limited to using a single sacral marker to estimate COM position. Because the true COM is located anterior to the sacrum, the use of the sacral marker led to a systematic bias in our COM estimates. In addition, our COM estimates did not account for the influence of forward trunk tilt. It seems unlikely, however, that these limitations had a impact on our findings, as the systematic COM bias would tend to affect all task conditions equally and the task-related differences in forward trunk tilt angle were small (a few degrees, on average). If anything, the present findings likely underestimate the effect of tread length. During stair descent, forward trunk tilt would bring the true COM further forward and thereby reduce stability margins; hence, the tendency for forward trunk tilt to increase as tread length was reduced would tend to exacerbate the reduction in stability margin that was found to occur at the shorter tread lengths.

\section{Conclusion}

In summary, we have demonstrated a significant impact of run length and riser height on dynamic balance control in the sagittal plane during stair descent and differential effects of 
aging. Since we did not find any interaction effects between rise and run, this demonstrates that there is not a combination of riser height and run length which impacts balance control to a significant degree. Rather, addressing each component of stair design individually is important to facilitate dynamic balance control for both older and young adults. The findings help to explain underlying biomechanical factors associated with increased risk of falls during stair descent and the relationship with step geometry. Considering the importance of stair ambulation in maintaining independence and activity in the community, this study highlights the definite need for safer stair design standards to minimize the risk of falls and increase stair safety across the lifespan.

\section{Acknowledgments}

The authors would like to acknowledge financial support from the Canadian Institutes of Health Research Post Doctoral Fellowship (AC Novak), Canadian Institutes of Health Research Operating Grant (CIHR MOP 130519), National Institute on Disability and Rehabilitation Research (NIDRR) through the Rehabilitation Engineering Research Center on Universal Design and the Built Environment (a partnership with the Center for Inclusive Design and Environmental Access - grant \# H133E100002), and an Ontario Graduate Scholarship (V Komisar). The authors would also like to acknowledge technical assistance of Mr. Peter Goshulak.

\section{Conflict of Interest}

The authors declare they have no conflict of interest 


\section{References}

1. Tromp AM, Pluijm SM, Smit JH, Deeg DJ, Bouter LM, Lips P. Fall-risk screening test: a prospective study on predictors for falls in community-dwelling elderly. J Clin Epidemiol 2001; 54: 837-844.

2. Arfken CL, Lach HW, Birge SJ, Miller JP. The prevalence and correlates of fear of falling in elderly persons living in the community. Am J Public Health 1994; 84: 565570.

3. Howland J, Lachman ME, Peterson EW, Cote J, Kasten L, Jette A. Covariates of fear of falling and associated activity curtailment. Gerontologist 1998; 38: 549-555.

4. Tinetti ME, Mendes de Leon CF, Doucette JT, Baker DI. Fear of falling and fall-related efficacy in relationship to functioning among community-living elders. J Gerontol 1994; 49: M140-147.

5. Brouwer B, Musselman K, Culham E. Physical function and health status among seniors with and without a fear of falling. Gerontology 2004; 50: 135-141.

6. Cumming RG, Salkeld G, Thomas M, Szonyi G. Prospective study of the impact of fear of falling on activities of daily living, SF-36 scores, and nursing home admission. $J$ Gerontol A Biol Sci Med Sci 2000; 55: M299-305.

7. Startzell JK, Owens DA, Mulfinger LM, Cavanagh PR. Stair negotiation in older people: a review. J Am Geriatr Soc 2000; 48: 567-580.

8. Roys MS. Serious stair injuries can be prevented by improved stair design. Appl Ergon 2001; 32: 135-9.

9. Soriano TA, DeCherrie LV, Thomas DC. Falls in the community-dwelling older adult: A review for primary care providers. Clin Interv Aging 2007; 2: 545-553. 
10. Bosse I, Oberlander KD, Savelberg KD, Meijer K, Bruggemann GP, Karamanidis K. Dynamic stability control in younger and older adults during stair descent. Hum Mov Sci 2012; 31: 1560-70.

11. Hamel KA, Okita N, Higgingson JS, Cavanagh PR. Foot clearance during stair descent:effects of age and illumination. Gait Posture 2005; 21: 135-40.

12. Nadeau S, McFadyen BJ, Malouin F. Frontal and sagittal plane analyses of the stair climbing task in healthy adults aged over 40 years: what are the challenges compared to level walking? Clin Biomech (Bristol, Avon) 2003; 18: 950-9.

13. Novak AC, Brouwer B. Sagittal and frontal lower limb joint moments during stair ascent and descent in young and older adults. Gait Posture 2011; 33: 54-60.

14. Novak AC, Li Q, Yang S, Brouwer B. Mechanical energy transfers across lower limb segments during stair ascent and descent in young and healthy older adults. Gait Posture 2011; 34: 384-90.

15. Reid SM, Graham RB, Costigan PA. Differentiation of young and older adult stair climbing gait using principal component analysis. Gait Posture 2010; 31: 197-203.

16. Reid SM, Novak AC, Brouwer B, Costigan PA. Relationship between stair ambulation with and without a handrail and centre of pressure velocities during stair ascent and descent. Gait Posture 2011; 34: 529-32.

17. Simoneau GG, Cavanagh PR, Ulbrecht JS, Leibowitz HW, Tyrrell RA. The influence of visual factors on fall-related kinematic variables during stair descent by older women. $J$ Gerontol 1991; 46: M188-95. 
18. Zachazewski JE, Riley PO, Krebs DE. Biomechanical analysis of body mass transfer during stair ascent and descent of healthy subjects. J Rehabil Res Dev 1993; 30: 412-22.

19. Zietz D, Hollands M. Gaze behavior of young and older adults during stair walking. $J$ Mot Behav 2009; 41: 357-65.

20. Novak AC, Brouwer B. Strength and aerobic requirements during stair ambulation in persons with chronic stroke and healthy adults. Arch Phys Med Rehabil 2012; 93: 683689.

21. Tse T. The environment and falls prevention: Do environmental modifications make a difference? Aust Occupational Therapy Journal 2005; 52: 271-281.

22. Wright M, Roys MS. Accidents on English stairs are directly related to going size, in Contemporary Ergonomics, P.D. Bust, Editor. 2008, Taylor and Francis: London. 632637.

23. Wright M, Roys MS. Effect of changing stair dimensions on safety, in Contemporary Ergonomics, P.D. Bust and P.T. McCabe, Editors. 2005, Taylor and Francis: London. 469-474.

24. Riener R, Rabuffetti M, Frigo C. Stair ascent and descent at different inclinations. Gait Posture 2002; 15: 32-44.

25. Templer J. The Staircase: studies of hazards, falls and safer design. 1992, The MIT Press: Cambridge, Massachusetts.

26. Stacoff A, Diezi C, Luder G, Stussi E, Kramers-de Quervain IA. Ground reaction forces on stairs: effects of stair inclination and age. Gait Posture 2005; 21: 24-38. 
27. Yang F, Pai YC. Can sacral marker approximate center of mass during gait and slip-fall recovery among community-dwelling older adults? J Biomech 2014; 18: 3807-3812.

28. Miyasike-daSilva V, Allard F, Mcllroy WE. Where do we look when we walk on stairs?Gaze behaviour on stairs, transitions, and handrails. Exp Brain Res 2011; 209: 7383.

29. Cluff T, Robertson DG. Kinetic analysis of stair descent: Part 1. Forwards step-over-step descent. Gait Posture 2011; 33: 423-8.

30. Hof AL, Gazendam MG, Sinke WE. The condition for dynamic stability. $J$ Biomech $2005 ; 38: 1-8$.

31. Johnson DA, Pauls J. Systematic stair step geometry defects, increased injuries and public health plus regulatory responses, in Contemporary Ergonomics, A. M, Editor. 2010, CRC Press. 453-461.

32. Cohen J, LaRue CA, Cohen HH. An ergonomic analysis of 80 cases. Professional Safety 2009; 54: 27-32.

33. Novak AC, Deshpande N. Effects of aging on whole body and segmental control while obstacle crossing under impaired sensory conditions. Hum Mov Sci 2014; 35: 121-130.

34. Reeves ND, Spanjaard M, Mohagheghi AA, Baltzopoulos V, Maganaris CN. The demands of stair descent relative to maximum capacities in elderly and young adults. $J$ Electromyogrp Kinesiol 2008; 18: 218-227.

35. Buckley JG, Cooper G, Maganaris CN, Reeves ND. Is stair descent in the elderly associated with periods of high centre of mass downward accelerations? Exp Gerontol 2013; 48: 283-289. 
36. Grabiner MD, Donovan S, Bareither ML, Marone JR, Hamstra-Wright K, Gatts S, Troy KL. Trunk kinematics and fall risk of older adults: translating biomechanical results to the clinic. J Electromyogrp \& Kinesiol 2008; 18: 197-204.

37. Mian OS, Thom JM, Narici MV, Baltzopoulos V. Kinematics of stair descent in young and older adults and the impact of exercise training. Gait Posture 2007; 25: 9-17.

38. Antonio PJ, Perry SD. Quantifying stair gait stability in young and older adults, with modifications to insole hardness. Gait Posture 2014; 40: 429-434.

39. Zietz D, Johannsen L, Hollands M. Stepping characteristics and centre of mass control during stair descent: effects of age, fall risk and visual factors. Gait Posture 2011; 34: 279-284.

40. Buckley JG, Heasley K, Scally A, Elliot DB. The effects of blurring vision on mediolateral balance during stepping up or down to a new level in the elderly. Gait Posture 2005; 22: 146-153.

41. Novak AC, Brouwer B. Kinematic and kinetic evaluation of the stance phase of stair ambulation in persons with stroke and healthy adults: a pilot study. J Appl Biomech 2013; 29: 443-452. 


\section{Figure Captions}

Figure 1. Custom-built stairs highlighting the maximum (left panel) and minimum (middle panel) run lengths used for the study. A handrail (not present in the figure) was present for all testing. However, participants were instructed to negotiate the stairs without the handrail. The figure on the far right panel depicts the definitions of the riser height and run length.

Figure 2. Forward upper body tilt angle for the transition step across run lengths (A) and riser heights (C) and during steady state stair descent, across run lengths (B) and riser heights (D). Positive angle represents forward flexion. Standard error bars are depicted on the figures

* indicates significant main effect of run length

† indicates significant interaction effect (age-by-rise)

Figure 3. Lateral upper body tilt angle for the transition step, across run lengths (A) and riser heights (C) and during steady state stair descent, across run lengths (B) and riser heights (D). Standard error bars are depicted on the figures

${ }^{\alpha}$ indicates significant main effect of age

Figure 4. Margin of stability $(\mathrm{cm})$ for the transition step, across run lengths $(\mathrm{A})$ and riser heights (C) and during steady state stair descent, across run lengths (B) and riser heights (D). Standard error bars are depicted on the figures

* indicates significant main effect of run length

$\uparrow$ indicates significant main effect of riser height 
${ }^{\alpha}$ indicates significant main effect of age

Figure 5. Velocity of the centre of mass (vCOM) for the transition step, across run lengths (A) and riser heights (C) and during steady state stair descent, across run lengths (B) and riser heights (D). Positive values represent forward velocity of the COM. Note the break in the vertical axis scale for more convenient visualization of data $(C, D)$. Standard error bars are depicted on the figures.

* indicates significant main effect of run length

${ }^{\alpha}$ indicates significant main effect of age

Figure 6. Position of the centre of mass relative to the step edge (dCOM) for the transition step across run lengths (A) and riser heights (C) and during steady state stair descent, across run lengths (B) and riser heights (D). Positive values represent the vertical projection of the COM positioned behind the step edge. Standard error bars are depicted on the figures.

* indicates significant main effect of run length

${ }^{\dagger}$ indicates significant main effect of age 
Tables

Table 1. Subject characteristics

\begin{tabular}{|c|c|c|c|c|c|c|}
\hline & \multicolumn{3}{|l|}{ Young adults } & \multicolumn{3}{|l|}{ Older adults } \\
\hline & All & Male & Female & All & Male & Female \\
\hline $\begin{array}{l}\text { \# of } \\
\text { participants }\end{array}$ & 14 & 8 & 6 & 14 & 7 & 7 \\
\hline Age (years) & $25.5 \pm 3.2$ & $26.4 \pm 2.8$ & $24.2 \pm 3.7$ & $73.1 \pm 6.0$ & $70.8 \pm 2.8$ & $75.14 \pm 7.5$ \\
\hline Weight (kg) & $72.0 \pm 15.1$ & $79.8 \pm 13.9$ & $59.5 \pm 6.1$ & $69.9 \pm 18.9$ & $84.6 \pm 16.7$ & $57.2 \pm 8.8$ \\
\hline Height (m) & $1.74 \pm .1$ & $1.8 \pm .1$ & $1.7 \pm .1$ & $1.67 \pm .1$ & $1.74 \pm .1$ & $1.59 \pm .1$ \\
\hline
\end{tabular}

Table 2. Imperial to metric conversions for all rise and run step dimensions tested

\begin{tabular}{lll}
\hline & Imperial measurements (inches) & Metric measurements (mm) \\
\cline { 2 - 3 } Run length & 8 & 203 \\
& 9 & 229 \\
& 10 & 254 \\
& 11 & 280 \\
& 12 & 305 \\
Riser height & 13 & 330 \\
& 14 & 356 \\
\hline \multirow{3}{*}{ tis. } & 7.5 & 178 \\
& 8 & 190 \\
\hline
\end{tabular}


Figure 1.

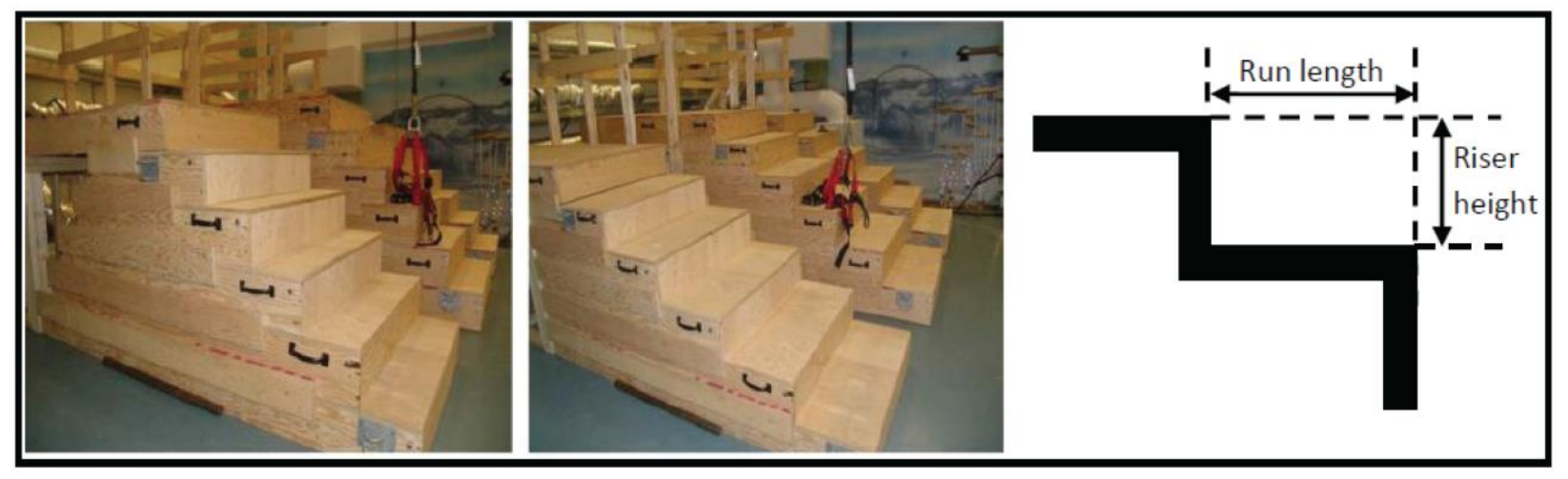


Figure 2.
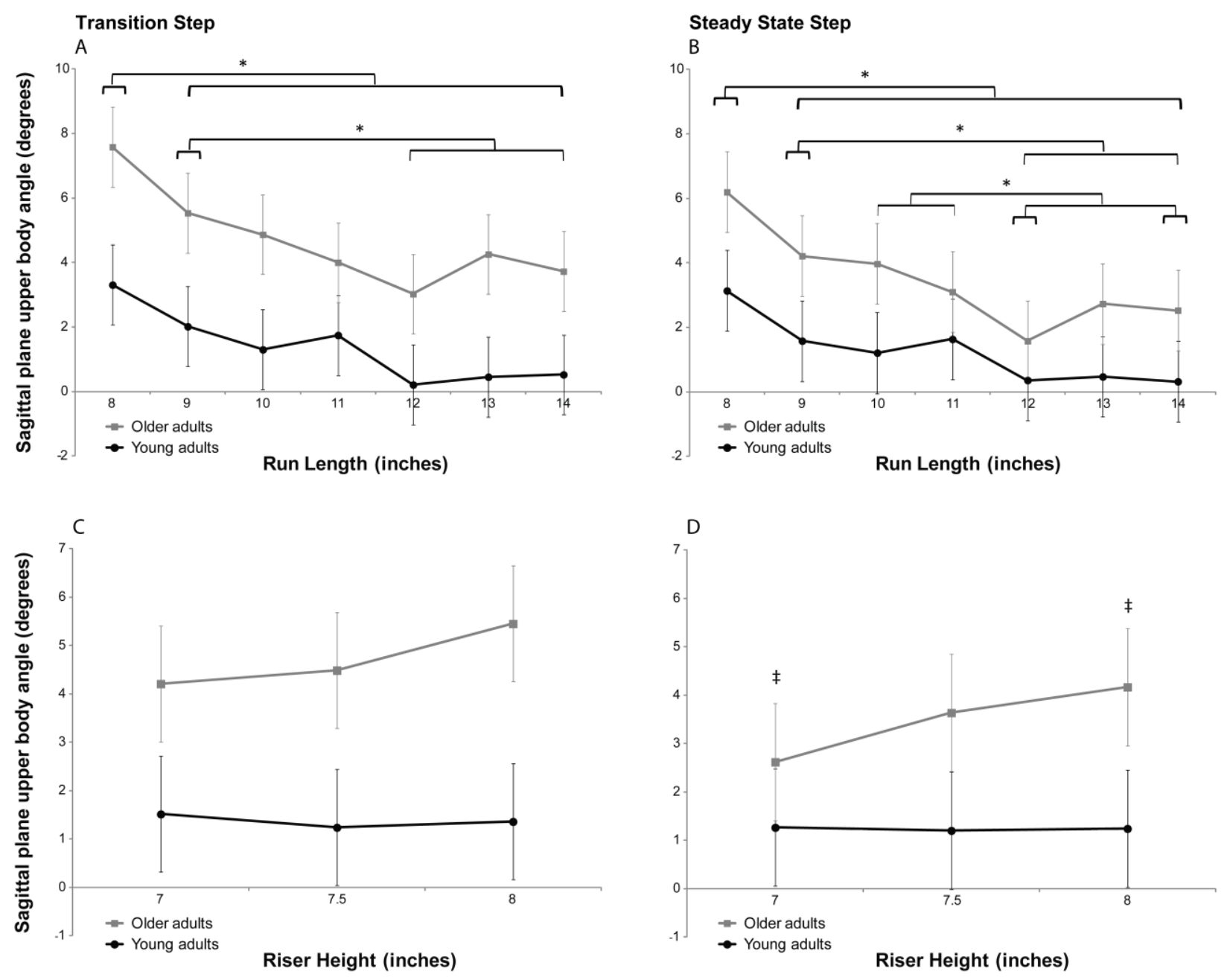
Figure 3.

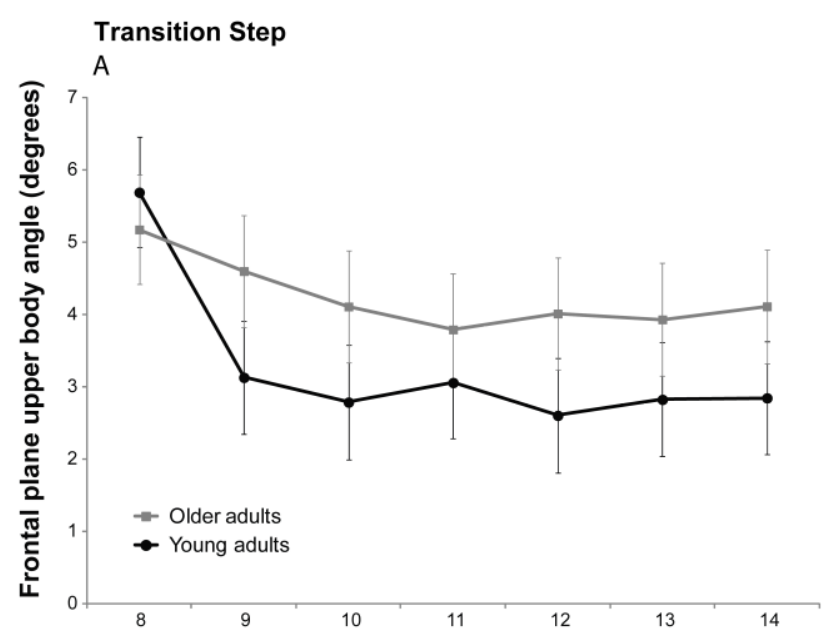

Run Length (inches)

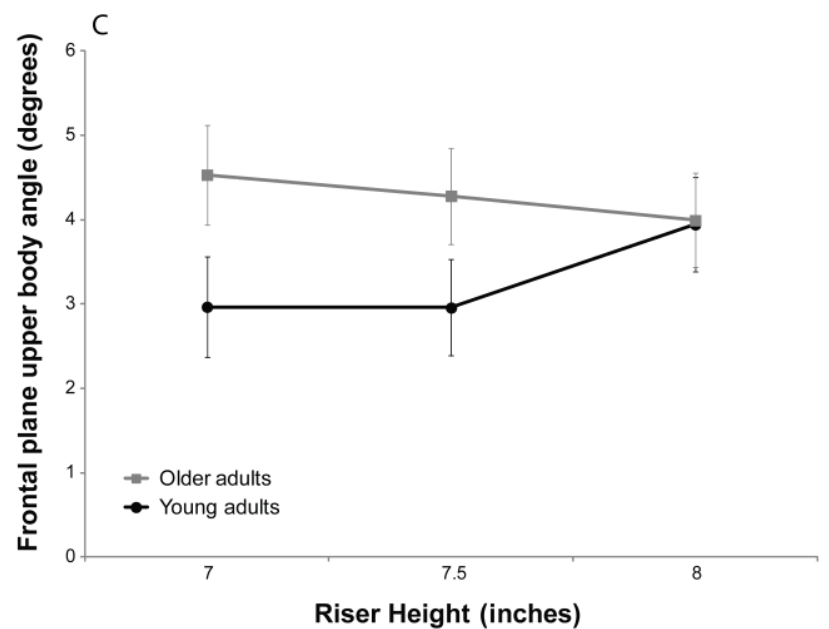

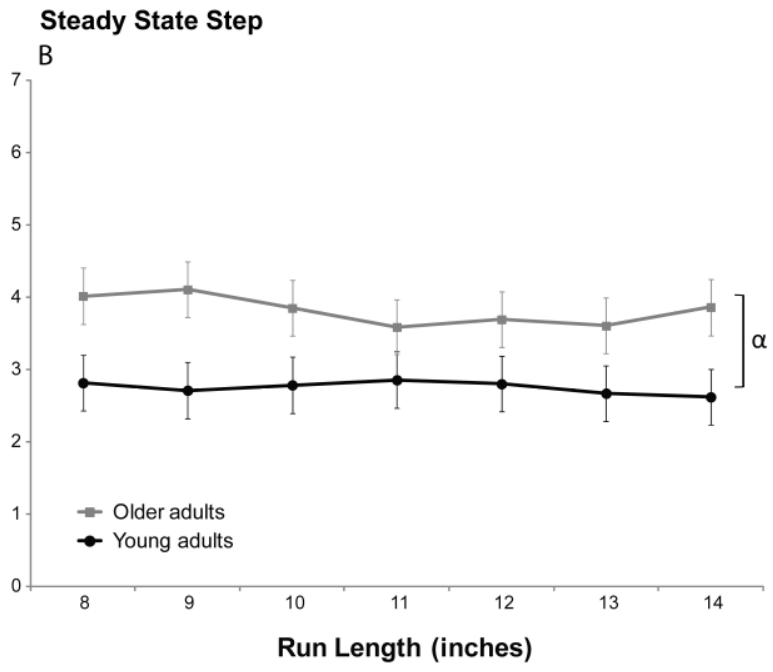

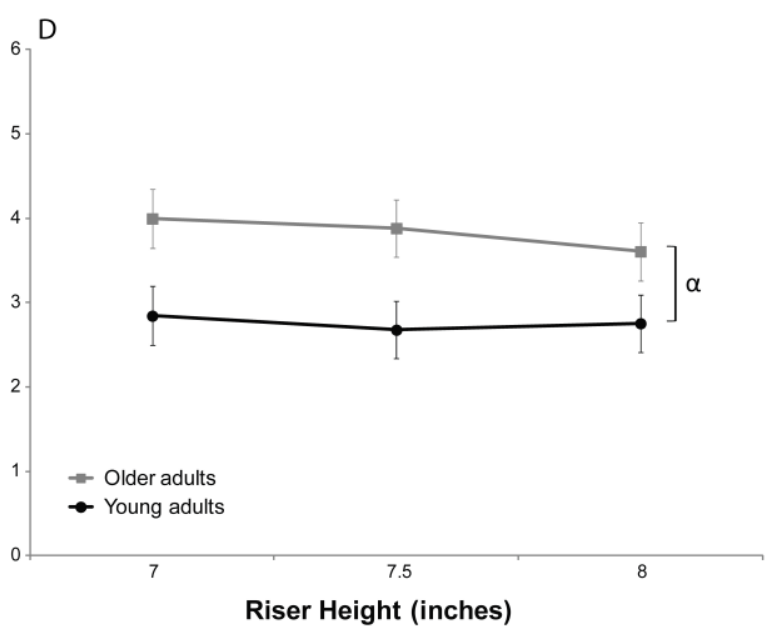


Figure 4.
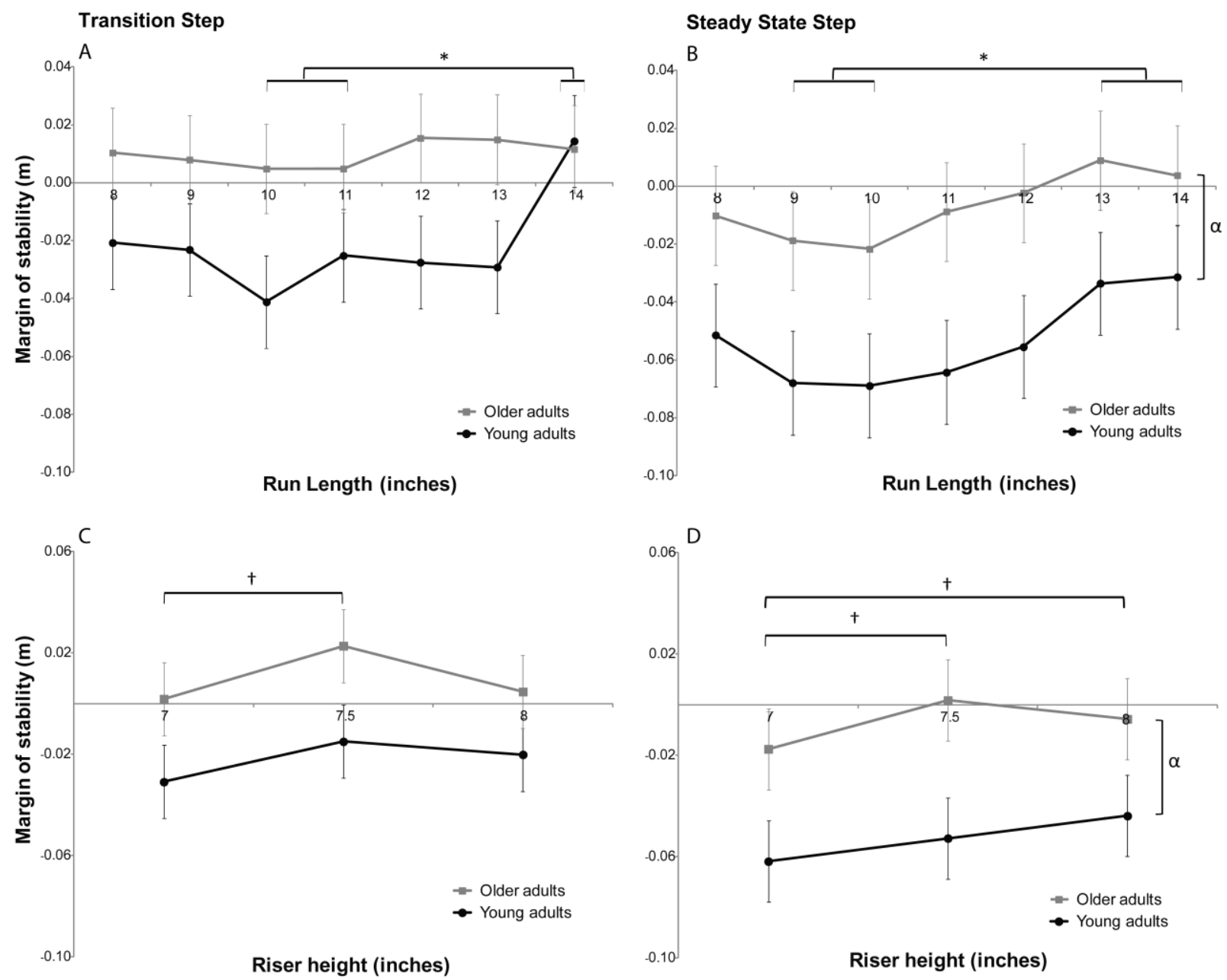
Figure 5.
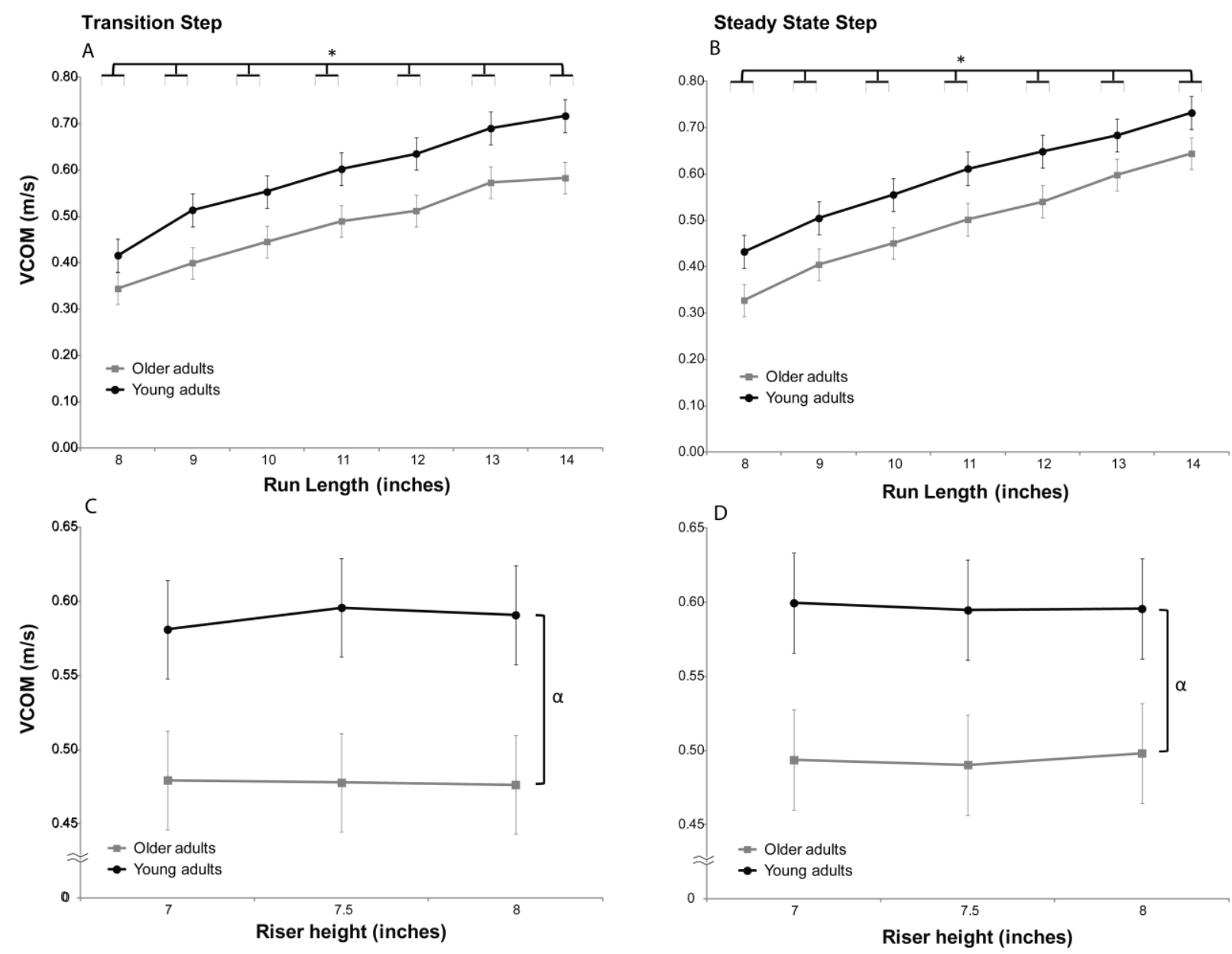
Figure 6.
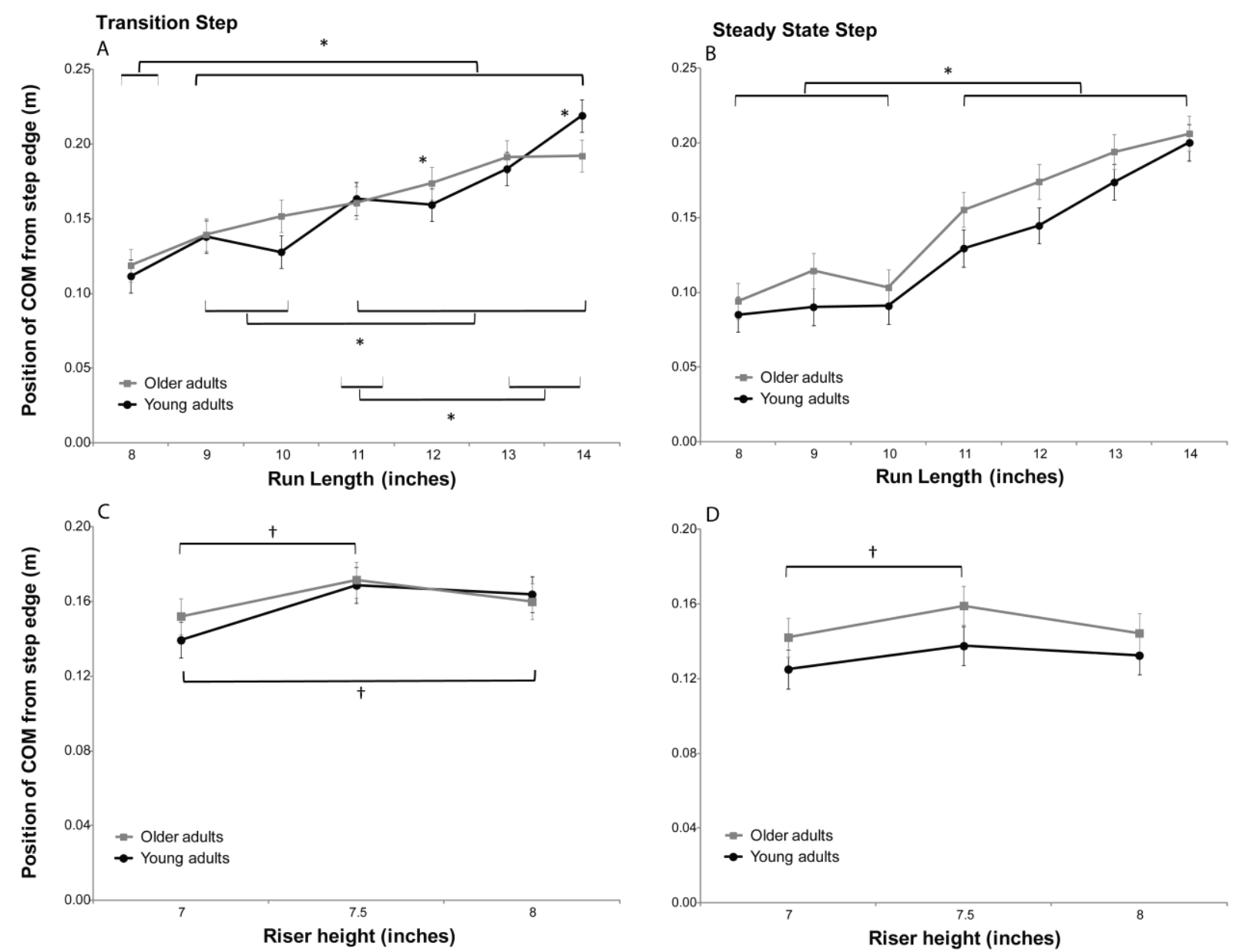